\title{
Addendum
}

\section{A functional limit theorem for Erdös and Rényi's law of large numbers}

\author{
Gabriela R. Sanchis \\ Department of Mathematical Sciences, Elizabethtown College, \\ Elizabethtown, PA 17022, USA
}

Probab. Theory Relat. Fields 98, 1-5 (1994)

Professor Paul Deheuvel has kindly called my attention to the fact that Theorem 1.1 in my paper was obtained in a more general form by Borovkov [2], who proves that, given an i.i.d. sequence $\left\{X_{n}\right\}$ of $\mathfrak{R}^{d}$-valued random vectors having finite moment-generating function, 0 expectation, and a nonsingular covariance matrix, then for any $c>0, \lim _{N \rightarrow \infty} h\left(\mathscr{H}_{N}, L_{c}\right)=0$ a.s., where $h(A, B)$ is the Hausdorff distance between sets $A, B \subset C^{d}[0,1]$ $=\left\{x:[0,1] \rightarrow \Re^{d} \mid x\right.$ is continuous and $\left.x(0)=0\right\}$, endowed with the sup norm, and $\mathscr{H}_{N}$ and $L_{c}$ are defined as in [1]; my paper deals only with the onedimensional case. Moreover, in [4], P. Deheuvel extends this result to the case where the moment generating function of the $X_{n}$ is not finite everywhere.

In addition, I should mention that the original version (1970) of the Erdös-Rényi theorem restricted the values of $c$; P. Deheuvel and L. Devroye [3] were first to give a full form of the Erdös-Rényi theorem, valid for each $c>0$.

\section{References}

1. Sanchis, G.R.: A functional limit theorem for Erdös and Rényi's law of large numbers. Probab. Theory Relat. Fields 98, 1-5 (1994)

2. Borovkov, K.A.: A functional form of the Erdös-Rényi law of large numbers. Theory Probab. App. 35, 762-766 (1990)

3. Deheuvels, P., Devroye, L.: Limit laws of Erdös-Rényi-Shepp type. Ann. Probab. 15, 1363-1386 (1987)

4. Deheuvels, P.: Functional Erdös-Rényi laws. Stud. Sci. Math. Hung. 26, 261-295 (1991) 